\title{
The role of microfluidics in protein formulations with pre-programmed functional characteristics
}

This article was published in the following Dove Press journal:

Biologics:Targets and Therapy

\section{Hu Meng ${ }^{1,2, *}$ \\ Shuai Deng ${ }^{1,2, *}$ \\ Yajing You ${ }^{3}$ \\ Hon Fai Chan ${ }^{1,2}$}

'Institute for Tissue Engineering and Regenerative Medicine, The Chinese University of Hong Kong, Hong Kong, China; ${ }^{2}$ School of Biomedical Sciences, The Chinese University of Hong Kong, Hong Kong, China; ${ }^{3}$ Department of Immunology, School of Basic Medical Sciences, Shandong University, Jinan, China

*These authors contributed equally to this work
Correspondence: Hon Fai Chan Institute for Tissue Engineering and Regenerative Medicine, I07A, Lo KweeSeong Integrated Biomedical Sciences Building, Area 39, The Chinese University of Hong Kong, Shatin, N.T., Hong Kong, China

Tel +85239433032

Email honfaichan@cuhk.edu.hk

\begin{abstract}
Protein-based therapies hold great promise for treating many diseases. Nevertheless, the challenges of producing therapies with targeted attributes via standardized processes may hinder the development of protein formulations and clinical translation of the advanced therapies. Microfluidics represents a promising technology to develop protein formulations with pre-programmed functional characteristics, including size, morphology, and controlled drug release property. In this review, we discuss some examples of adopting microfluidics for fabricating particle- and fiber/tube-based formulations and highlight the advantages of microfluidics-assisted fabrication.
\end{abstract}

Keywords: protein therapy, microfluidics, biofabrication, controlled release

\section{Introduction}

Protein-based therapies have been rapidly developed to treat many diseases, such as cancer and metabolic and autoimmune disorders. ${ }^{1}$ The delivery of proteins such as growth factor can also have significant impact in the field of regenerative medicine. Globally, the market of protein-based therapy is expanding dramatically, which was valued at around $\$ 174.7$ billion in 2015 and is expected to reach over $\$ 240$ billion by $2020 .^{2}$ Examples of protein products include monoclonal antibodies and fusion protein..$^{3,4}$

In general, protein drugs show higher binding selectivity and specificity to target molecules with low toxicity compared to small-molecule drugs. ${ }^{5}$ However, they often suffer from low bioavailability due to degradation, aggregation, adsorption, and denaturation. ${ }^{6}$ To enhance the performance of protein formulations, functional characteristics can be programmed during the preparation steps. These characteristics include size, morphology, and controlled drug release property.

To effectively deliver drugs to target site, micro-/nanoparticles encapsulating protein drugs have been developed, offering protection against premature degradation as well as serving as drug depot for controlled release of protein. ${ }^{7}$ Proteins can also be engineered into different configurations, such as fiber and tube, which can be applied in various drug delivery and tissue engineering applications. ${ }^{8,9}$ Nevertheless, the lack of advanced fabrication approaches that facilitate the development of protein formulations with controllable attributes significantly hinders clinical translation of protein-based formulations. ${ }^{10}$

The Food and Drug Administration (FDA) has issued the guidelines of current Good Manufacturing Practices (cGMP) for biomanufacturing to ensure products 
(eg, drugs) are fabricated with the desired characteristics in terms of identity, strength, quality, and purity. ${ }^{11}$ The use of microfluidics, which refers to the manipulation of fluid flow in tiny scale (micro- or pico-liter), to fabricate biologically relevant materials is a promising approach owing to the multiple advantages it offers. ${ }^{12}$ Here, we review the rationale and examples of adopting microfluidics for the fabrication of protein formulations with pre-programmed functional characteristics and highlight the role of microfluidics in advancing clinical translation of protein-based therapies.

\section{Introduction of microfluidic technologies}

Microfluidics is a technology characterized by the study and manipulation of fluids at the submillimeter scale and has shown great promise in biomedical applications. ${ }^{13}$ The core of the technology is a device with channels of submillimeter scale and fluid flow in the device is driven by pump or manual injection. Due to the small dimension of the channel, the Reynolds number, which represents the relative importance of inertia to viscous force, is small (typically between $10^{-6}$ and 10) and hence laminar flow often results. ${ }^{14}$ This allows better control over molecules/particles distribution in fluidic system. One distinction between microscale and macroscale fluid phenomena is that the relative effects of various forces are different. Surface tension and capillary forces are dominant at the microscale, whereas the effect of gravity is less important as the dimension becomes smaller. This facilitates operations such as generating monodisperse emulsion droplets and patterning cells on surface. The interfacial flow is especially crucial in the case of droplet generation. ${ }^{14}$ Emulsion droplets are generated when two or more immiscible streams such as water and oil, in the presence of a surfactant, are injected into a microfluidic channel in various configurations. Some examples of device configuration are T-junction, flow-focusing, and concentric flow. ${ }^{15}$ These emulsion droplets serve as uniform-sized reactor to compartmentalize the bulk reaction mixture into discrete and controllable units. This can lead to consistent material fabrication. The mode of droplet formation is determined by the ratio of viscous force in the continuous phase to the interfacial tension (ie, capillary number). ${ }^{16}$ Besides, active techniques such as electrical, magnetic, centrifugal, and mechanical inputs can also be introduced to further manipulate the droplet formation. ${ }^{14}$ Significant effort has been devoted to study the physical mechanisms of droplet formation, which was reviewed elsewhere. ${ }^{17}$

In general, microfluidic system is associated with benefits such as sample volume reduction, device miniaturization, rapid heat and mass transfer, amenability to scaling up and high-throughput screening, and enhanced processing accuracy and efficiency. ${ }^{18-20}$ The amount of reagent input and device footprint can be reduced in microfluidic operation compared with conventional macroscale bioprocessing. ${ }^{21}$ This can lead to reduced cost and better controllability. In addition, the physical properties of resulting particle formulation can be easily tuned by adjusting the total flow rate, polymer concentration, aqueous-to-organic flow rate ratio, the use of a co-solvent, and the rate of solvent mixing. ${ }^{22}$ Protein therapeutics created by microfluidic approaches can be categorized into two main types: particle-based and fiber/tube-based formulations (Figure 1). We will examine each category and discuss how microfluidics contributes to programing functional characteristics of protein.

\section{Particle-based formulation}

\section{Polymeric micro/nanoparticle as protein carrier}

Since protein therapeutics are very sensitive to enzymatic degradation, aggregation, adsorption, and denaturation and they usually target specific sites of the body, encapsulating them in carrier can protect them from degradation and delivering them to target site via surface modification (in the case of nanoparticle (NP)). The first US FDA-approved protein delivery therapy was polyethylene glycol(PEG)ylated adenosine deaminase (Adagen $^{\circledR}$; Leadiant Biosciences, Pomezia, Italy) in 1990. Since then, various classes of carriers for protein delivery have been developed..$^{15}$ Examples of nanoscale carriers include lipid-based (eg, liposome and exosome) and inorganic (eg, calcium phosphate and mesoporous silica) NPs. The NPs can shield protein from denaturation and prolong circulation in the blood stream. ${ }^{23}$ Unlike NPs that can be administered systematically, microscale carriers are usually administered locally at the target site or subcutaneously for sustained protein release via diffusion through surface pore or degradation of carrier material. ${ }^{24}$

Two common strategies of micro/nanoparticle formation are nanoprecipitation and emulsification. During nanoprecipitation, the reduction of the quality of the solvent in which the ingredient of NPs is present triggers particle formation. The change in solvent quality can be achieved by altering salt concentration, solubility conditions, $\mathrm{pH}$, etc. ${ }^{15}$ For instance, precursors of NP and protein can be dissolved in an aqueous solvent and added to another solvent with good water miscibility but as a poor solvent for the constituents. NPs will be precipitated out of the mixture. NPs synthesized in typical bulk condition are usually prone to polydispersity and batchto-batch variation due to longer timescale of solvent exchange (in the order of seconds) than that of precursor nucleation 


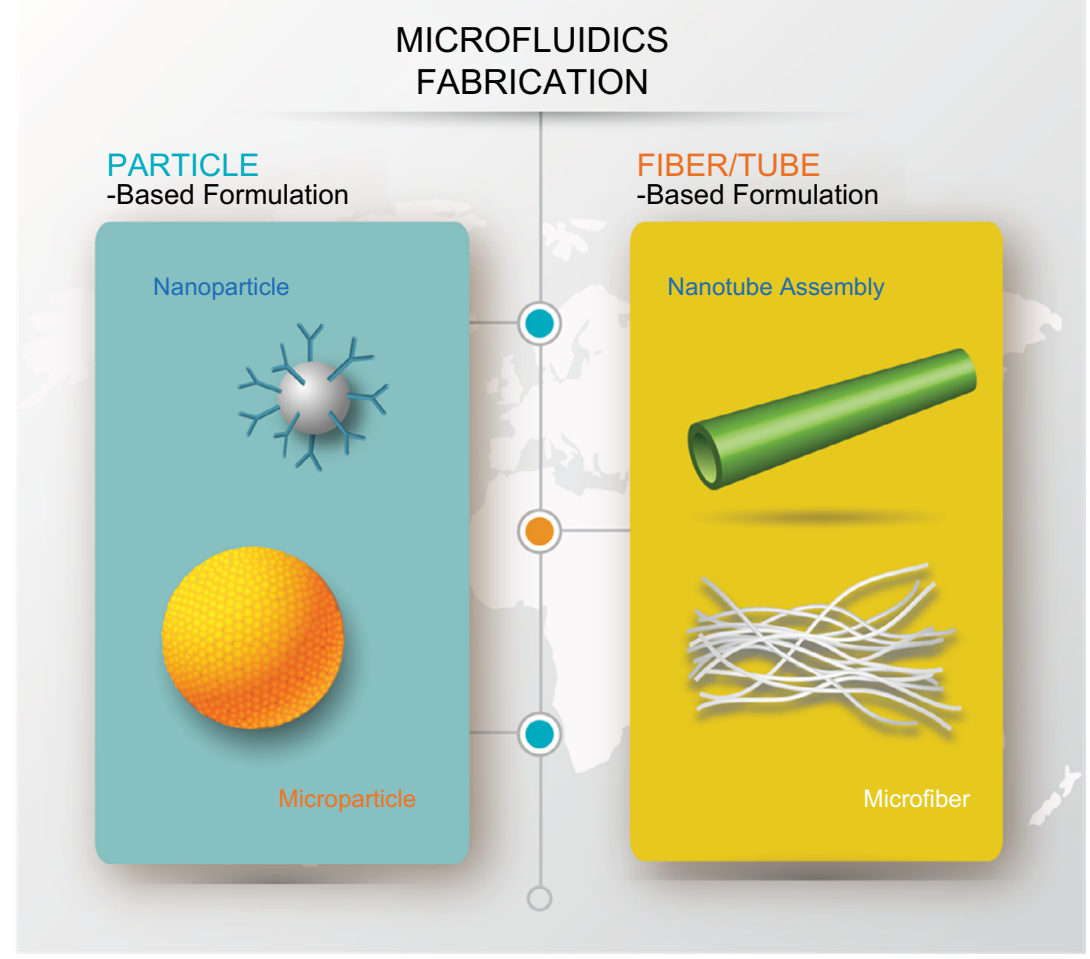

Figure I Illustration of different forms of protein formulation fabricated using microfluidics system.

and growth, resulting in uncontrolled NP aggregation. The use of microfluidics can improve the process by expediting solvent exchange via hydrodynamic flow focusing, where the precursor solution is focused into a narrow stream by two streams of anti-solvent flowing in parallel (Figure 2A). ${ }^{25}$ Rapid diffusion can occur through the interface. Vortex and turbulence created in high speed flow within hydrodynamic focusing can enable even shorter mixing time and increase the production rate. ${ }^{26,27} \mathrm{~A}$ recent report described the use of a Staggered Herringbone Mixer for rapid mixing of two streams and channeled to a Tangential Flow Filtration device for subsequent purification, which led to high-throughput liposome production and effective removal of non-entrapped protein (ovalbumin). ${ }^{28}$

Another common way of fabricating micro/nanoparticles is by emulsification followed by solvent depletion, such as evaporation and solvent diffusion..$^{15}$ Tiny emulsion droplets containing protein and particle precursor are first generated by vigorous agitation or mixing two immiscible phases. A subsequent mixing step can be applied to generate double emulsion for preparing core-shell structures. The droplet content will condensate and form micro/nanoparticles after solvent depletion. The final size of particles is directly related to the size of the emulsion droplet, which can be very heterogeneous in bulk mixing, thus resulting in uncontrollable drug release profile. The benefit of forming monodisperse emulsion droplet in a microfluidic platform can contribute significantly to the formation of monodisperse particles with higher encapsulation efficiency and more controlled drug release rate. ${ }^{29,30} \mathrm{Also}$, the precise introduction of reagents into the droplets could be realized using pulse width modulation as demonstrated in one report. ${ }^{31}$ The cell-free synthesis of green fluorescent proteins inside double emulsions was monitored and the influence of DNA concentration on protein produced could be evaluated. In another report, microparticles composed of poly(vinyl alcohol) loaded with bovine serum albumin were fabricated with a size range of $23-47 \mu \mathrm{m}$ and an encapsulation efficiency of $84 \% .{ }^{32}$ Another report described the fabrication of monodisperse silk fibroin microparticles as a drug carrier using microfluidic platform due to its good biocompatibility, lack of toxicity, and immunogenicity. ${ }^{33}$

Furthermore, growing evidence has shown that particle shape may influence the particle targeting, drug loading, and release properties..$^{34}$ For example, more hydrophobic drug molecules could be loaded into worm-like micelles than spherical ones due to the presence of larger core volume. ${ }^{35}$ 


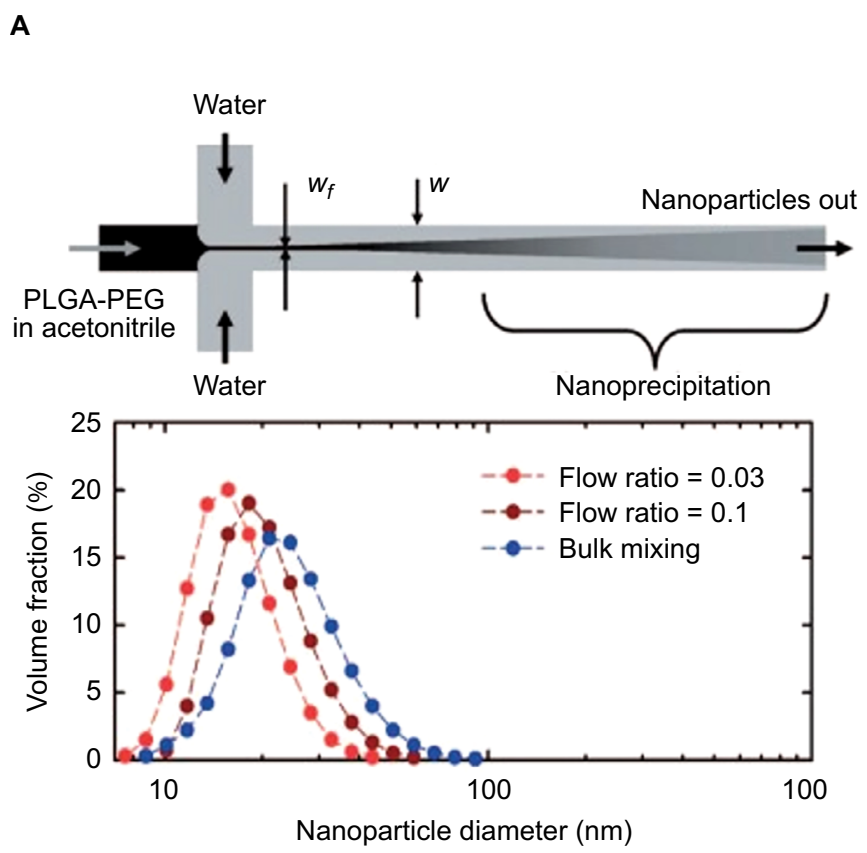

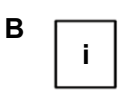
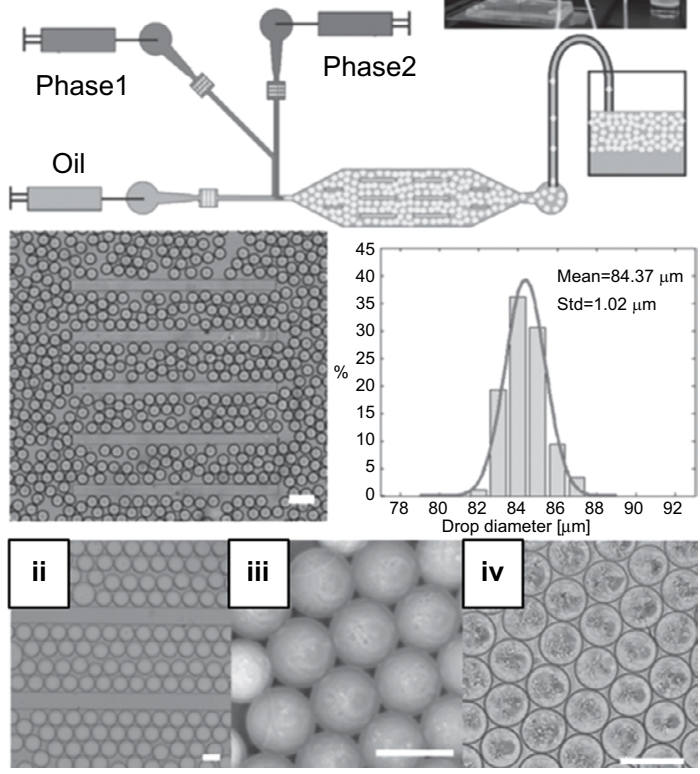

Figure 2 (A) Top: Schematic of formation of nanoparticle via hydrodynamic flow focusing. Bottom: The size distribution of nanoparticles fabricated by different approaches (flow ratio $=0.03$ and 0.1 refer to ratio of flow rates of PLGA-PEG/water). Reprinted (adapted) with permission from Karnik R, Gu F, Basto P, et al. Microfluidic platform for controlled synthesis of polymeric nanoparticles. Nano Lett. 2008;8(9):2906-2912.25 Copyright (2008) American Chemical Society. (B) (i) Schematic of formation of microfluidic emulsion droplets. Middle: Image of droplets produced and their size distribution. Scale bar is $200 \mu \mathrm{m}$. (ii) Images of droplets stored in the device, (iii) collected off-chip, and (iv) forming microparticles via self-assembly after 24 hours. Scale bar is $100 \mu \mathrm{m}$. Copyright @ 20I4. Dove Medical Press. Adapted from Bai S, Debnath S, Gibson $\mathrm{K}$, et al. Biocatalytic self-assembly of nanostructured peptide microparticles using droplet microfluidics. Small. 2014;10(2):285-293.39

Abbreviation: PLGA-PEG, poly(lactide-co-glycolide)-b-poly(ethylene glycol).

Based on the emulsification technique, the shape of the particle could be modulated by changing the geometry of the microchannel. ${ }^{36}$ Rod- and disc-like particles could be generated via controlled deformation of emulsion droplet inside the channel. Besides, a method that integrates microscope projection photolithography and microfluidics allowed continuous production of particles of distinct shapes. ${ }^{37}$ The monomer stream was infused through a microchannel above a mask containing desired features. Particles were polymerized when exposed to UV light through the mask and carried along by the stream for subsequent reaction to take place. The versatility of this approach enabled a wider variety of shapes to be produced for various applications.

\section{Self-assembly within emulsion droplet}

Self-assembly represents another strategy of formation of protein formulation, which involves the assembly of molecular building blocks into peptide- or protein-based particle. ${ }^{38} \mathrm{In}$ bulk, the process is usually conducted with minimal control over the macroscopic size and shape of the resulting particle. By confining building blocks into discrete, monodisperse emulsion droplets, we can exert better control over the particle properties. In one example, thermolysin and amino acid derivatives Fmoc-S (fluorenylmethyloxycarbony-serine) and F-OMe (phenylalanine methyl ester) were encapsulated in emulsion droplets for catalytic condensation to form microparticles (Figure 2B). The resulting spherulite microparticles were similar to those formed in bulk reaction but were more homogenous in size. ${ }^{39}$ In another example, emulsion droplet was used to study the coacervation of intrinsically disordered proteins by employing elastin-like polypeptides as model, where different coacervate architectures (ie, multilayer, mixed coacervate, and puncta) could be achieved by introducing a mixture of polypeptides with varying sequence, molecular weight, and concentration into the droplets. ${ }^{40}$ Overall, the use of emulsion droplet permits the study and control of individual microenvironment for self-assembly to occur.

\section{Fiber/tube-based formulation} Flow-based self-assembly

In addition to droplet-based system, self-assembly of peptide/ protein can also be controlled in a flow-based microfluidic device. For instance, the diphenylalanine building block would self-assemble into nanotubes in a spontaneous manner. By compartmentalizing nanotubes in microfluidic platform filled with micron-scale pillars and exposing them to building 
blocks of various concentrations, it is possible to manipulate the assembly process by controlling the net monomer influx at given time points and visualize the process (elongation and shortening) in real-time (Figure 3A). ${ }^{41}$ The control over the dimensional alteration will facilitate the design of supramolecular polymers such as protein formulation for various applications.

\section{Microfiber production}

Protein fibers are one of the major components of extracellular matrix, and some of them such as silk fiber are also found in nature. Micro- and nano-scale fibers hold great promise as implantable scaffold. They possess attributes such as short diffusion distance and high surface-to-volume ratio for mass exchange, thus they are suitable as cell culture platform and localized drug delivery vehicle. There are various ways to manufacture fibers, but not many of them are applicable to produce protein formulation. ${ }^{42}$ For example, melt spinning requires heating polymer above its melting point which can cause protein denaturation. Wet spinning, which is achieved by extruding material into a coagulation bath for solvent to diffuse out, often involves the use of harsh chemical that may not be optimal for protein application as well as for producing fiber with relatively a large diameter (20-200 $\mu \mathrm{m}) .^{43}$ Electrospinning is another technique that is commonly used to fabricate fibers, but the use of high voltage to draw the charged solution may preclude the loading of sensitive biological materials.

By using microfluidic flow focusing, microscale fibers can be generated with predictable and controllable properties. Through adjusting the flow rates of protein and surrounding buffer, fibers of various diameters can be fabricated. One
A

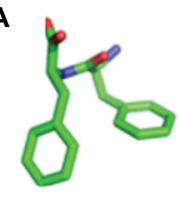

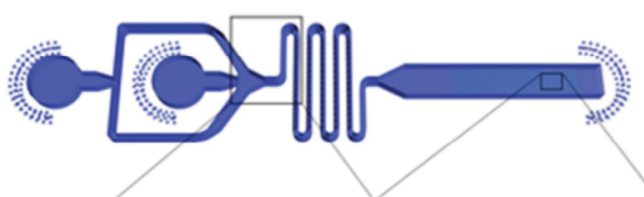

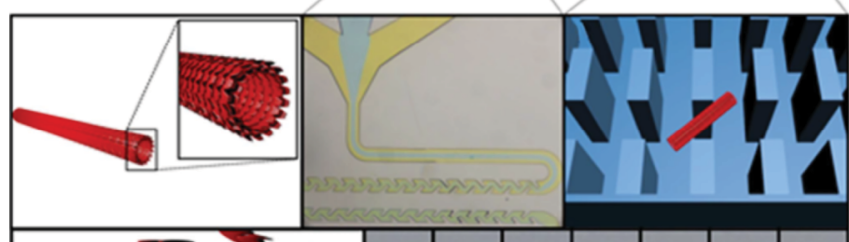

$\nabla$
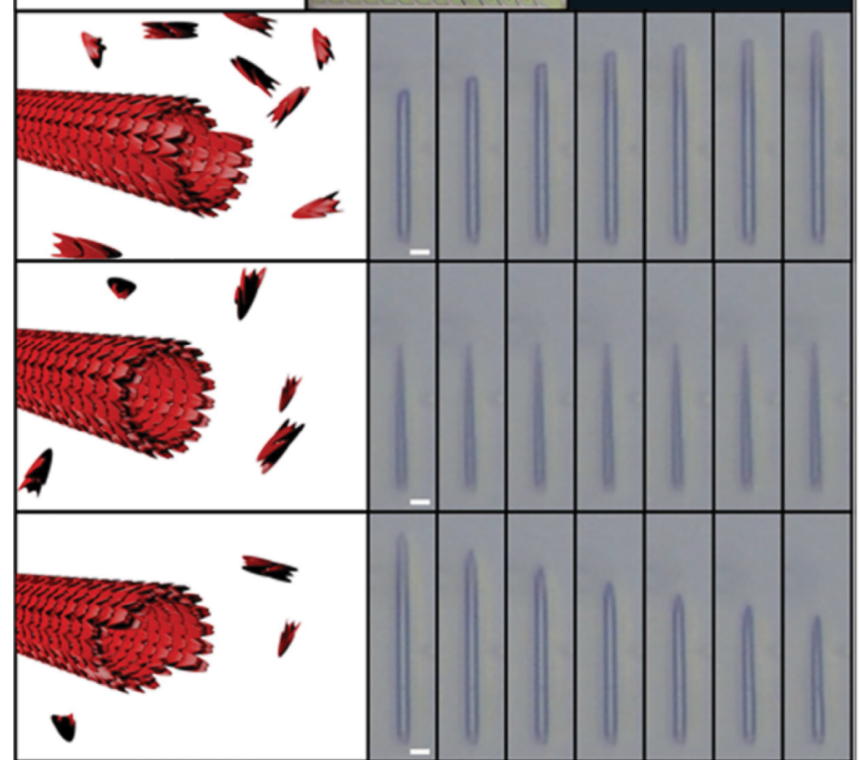

B
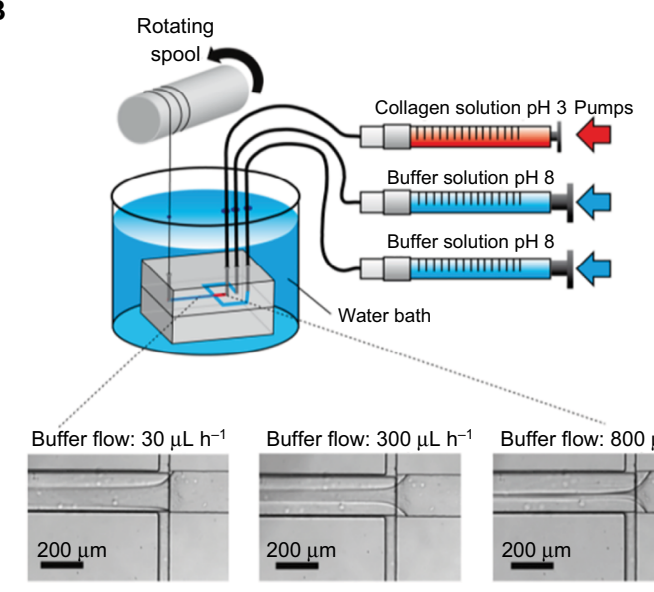

Buffer flow: $300 \mu \mathrm{L} \mathrm{h}$
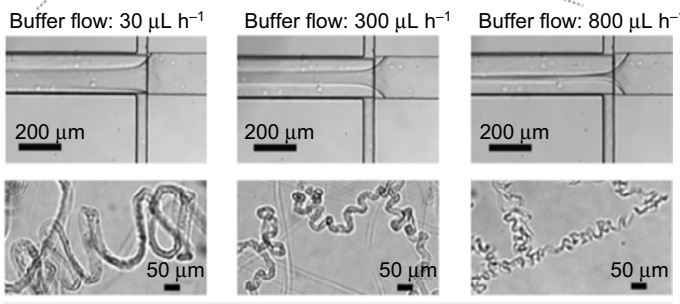

C

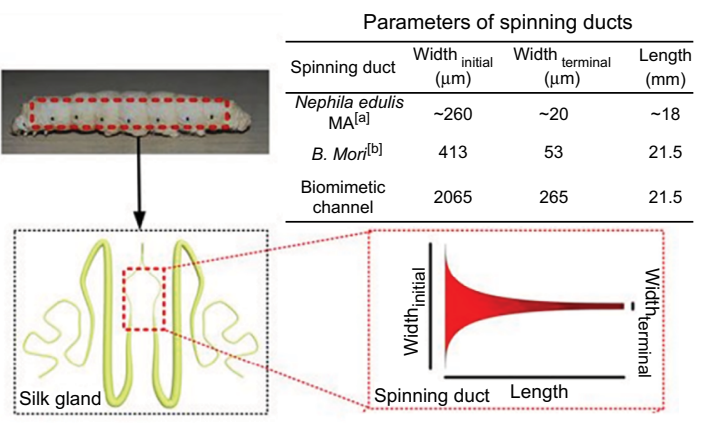

Figure 3 (A) Top: Diphenylalanine monomer and the design of the microfluidic device for nanotube assembly. Bottom: Illustration and imaging of nanotube formation at supercritical, critical, and subcritical monomer concentrations in flow. Scale bar is $5 \mu \mathrm{m}$. Adapted from Arnon ZA, Vitalis A, Levin A, et al. Dynamic microfluidic control of supramolecular peptide self-assembly. Nat Commun. 2016;7:13190. Creative Commons license and disclaimer available from: http://creativecommons.org/licenses/by/4.0/ legalcode. ${ }^{41}$ (B) Schematic of microfluidic chip for collagen microfiber formation and images of microfibers fabricated with varying buffer flow rates. Reprinted (adapted) with permission from Haynl C, Hofmann E, Pawar K, Förster S, Scheibel T. Microfluidics-produced collagen fibers show extraordinary mechanical properties. Nano Lett. 2016;16(9):5917-5922.43 Copyright (2008) American Chemical Society. (C) Schematic of the microfluidic spinning process of recombinant spider dragline silk and geometries of the spinning ducts of spider and silkworm. A biomimetic, microfluidic channel was designed to mimic the contracting geometry of spinning ducts to form compact and ordered silk protein microfiber. Adapted from Peng Q, Zhang Y, Lu L, et al. Recombinant spider silk from aqueous solutions via a bio-inspired microfluidic chip. Sci Rep. 2016;6:36473. Creative Commons license and disclaimer available from: http://creativecommons.org/licenses/by/4.0/legalcode. ${ }^{45}$ 
study reported that collagen fibers with diameters as small as $3 \mu \mathrm{m}$ could be produced (Figure $3 \mathrm{~B}$ ). ${ }^{43}$ Meanwhile, it has been observed that the silk gland in the silkworm and spider function similarly as a microfluidic system. ${ }^{44}$ The concentrated spinning dope would flow along a contracted gland and is exposed to solution with ionic gradients which regulate crystallization and protein aggregation. A biomimetic microfluidic device that mimics the specific geometry found in silkworm or spider or by means of flow focusing can reproduce the silk spinning process (Figure 3C). ${ }^{45}$ In addition, the shearing and elongational forces supplied by microfluidics also direct the alignment of $\beta$-sheets during fiber formation, resulting in fibers of tunable diameter, stiffness, and toughness. ${ }^{46,47}$ Compared with traditional wet spinning, microfluidic-based approach is more economical and nontoxic, which will facilitate the rapid and inexpensive fabrication of fiber of protein formulation.

\section{Future perspectives}

The demand for protein-based therapies is constantly rising, but fabricating protein formulations with controllable and pre-programmed functional characteristics is still a challenge. To comply with cGMP, technologies that enable controlled fabrication of protein formulation with pre-programmed functional characteristics must be developed. These characteristics include size, morphology, and controlled drug release property. Microfluidics has emerged as a potential platform to advance biofabrication of particle- and fiber/tube-based protein formulation. Here, we have reviewed several technologies that can not only improve the quality of protein formulations but also facilitate the design of supramolecular polymers for various protein applications. However, most of the previous reports were proof-of-concept studies and the translation of microfluidicbased formulation to preclinical and clinical application is still a challenge. One limitation is that microfluidic platform generally has a low production rate. To address this, efforts are devoted to scaling up the microfluidics technologies, such as by utilizing a microfluidic module with 128 cross-junctions that can produce droplets at a rate of $5.3 \mathrm{~mL} / \mathrm{min} .{ }^{48}$ Inspiration can also be drawn from the computer chip industry where massively parallel and miniaturized processing systems are developed to analyze big data. We envision that increased adoption of microfluidic technologies should pave the way for more effective protein-based therapy in the near future.

\section{Acknowledgment}

This work was supported by the Chinese University of Hong Kong start-up fund.

\section{Disclosure}

The authors report no conflicts of interest in this work.

\section{References}

1. Craik DJ, Fairlie DP, Liras S, Price D. The future of peptide-based drugs. Chem Biol Drug Des. 2013;81(1):136-147.

2. Dewan SS. Global Markets and Manufacturing Technologies for Protein Drugs (BIO021E). 2016. Available from: https://www.bccresearch.com/ report/download/report/bio021e. Accessed November 19, 2018.

3. Weidle UH, Schneider B, Georges G, Brinkmann U. Genetically engineered fusion proteins for treatment of cancer. Cancer Genom Proteom. 2012;9(6):357-372.

4. Chames P, van Regenmortel M, Weiss E, Baty D. Therapeutic antibodies: successes, limitations and hopes for the future. Br J Pharmacol. 2009;157(2):220-233.

5. Tiwari G, Tiwari R, Sriwastawa B, et al. Drug delivery systems: an updated review. Int J Pharm Invest. 2012;2(1):2-11.

6. Fix JA. Oral controlled release technology for peptides: status and future prospects. Pharm Res. 1996;13(12):1760-1764.

7. Langer R. Drug delivery and targeting. Nature. 1998;392(6679 Suppl):5-10.

8. Xu W, Yang Y. Drug sorption onto and release from soy protein fibers. J Mater Sci Mater Med. 2009;20(12):2477-2486.

9. Bruno BJ, Miller GD, Lim CS. Basics and recent advances in peptide and protein drug delivery. Ther Deliv. 2013;4(11):1443-1467.

10. Kobsa S, Saltzman WM. Bioengineering approaches to controlled protein delivery. Pediatr Res. 2008;63(5):513-519.

11. Federal Register Division. The Code of Federal Regulations of the United States of America. 1999. Available from: https://www.gpo.gov/ fdsys/pkg/FR-1999-01-08/content-detail.html. Accessed November 19, 2018.

12. Chan HF, Ma S, Leong KW. Can microfluidics address biomanufacturing challenges in drug/gene/cell therapies? Regen Biomater. 2016;3(2):87-98.

13. Whitesides GM. The origins and the future of microfluidics. Nature. 2006;442(7101):368-373.

14. Zhu P, Wang L. Passive and active droplet generation with microfluidics: a review. Lab Chip. 2016;17(1):34-75.

15. Zhang Y, Chan HF, Leong KW. Advanced materials and processing for drug delivery: the past and the future. Adv Drug Deliv Rev. 2013;65(1):104-120.

16. Zhu P, Kong T, Lei L, Tian X, Kang Z, Wang L. Droplet breakup in expansion-contraction microchannels. Sci Rep. 2016;6:21527.

17. Teh SY, Lin R, Hung LH, Lee AP. Droplet microfluidics. Lab Chip. 2008;8(2):198-220.

18. Ottino JM, Wiggins S. Introduction: mixing in microfluidics. Philos Trans A Math Phys Eng Sci. 2004;362(1818):923-935.

19. Chan HF, Zhang Y, Ho YP, Chiu YL, Jung Y, Leong KW. Rapid formation of multicellular spheroids in double-emulsion droplets with controllable microenvironment. Sci Rep. 2013;3:3462.

20. Chan HF, Zhang Y, Leong KW. Efficient one-step production of microencapsulated hepatocyte spheroids with enhanced functions. Small. 2016;12(20):2720-2730.

21. Song H, Chen DL, Ismagilov RF. Reactions in droplets in microfluidic channels. Angew Chem Int Ed Engl. 2006;45(44):7336-7356.

22. Khan IU, Serra CA, Anton N, Vandamme TF. Production of nanoparticle drug delivery systems with microfluidics tools. Expert Opin Drug Deliv. 2015;12(4):547-562.

23. de Jong WH, Borm PJ. Drug delivery and nanoparticles: applications and hazards. Int J Nanomedicine. 2008;3(2):133-149.

24. Kohane DS. Microparticles and nanoparticles for drug delivery. Biotechnol Bioeng. 2007;96(2):203-209.

25. Karnik R, Gu F, Basto P, et al. Microfluidic platform for controlled synthesis of polymeric nanoparticles. Nano Lett. 2008;8(9):2906-2912.

26. Valencia PM, Basto PA, Zhang L, et al. Single-step assembly of homogenous lipid-polymeric and lipid-quantum dot nanoparticles enabled by microfluidic rapid mixing. ACS Nano. 2010;4(3):1671-1679. 
27. Kim Y, Lee Chung B, Ma M, et al. Mass production and size control of lipid-polymer hybrid nanoparticles through controlled microvortices. Nano Lett. 2012;12(7):3587-3591.

28. Dimov N, Kastner E, Hussain M, Perrie Y, Szita N. Formation and purification of tailored liposomes for drug delivery using a module-based micro continuous-flow system. Sci Rep. 2017;7(1):12045.

29. Damiati S, Kompella UB, Damiati SA, Kodzius R. Microfluidic devices for drug delivery systems and drug screening. Genes. 2018;9(2):103.

30. Xu Q, Hashimoto M, Dang TT, et al. Preparation of monodisperse biodegradable polymer microparticles using a microfluidic flow-focusing device for controlled drug delivery. Small. 2009;5(13):1575-1581.

31. Chang JC, Swank Z, Keiser O, Maerkl SJ, Amstad E. Microfluidic device for real-time formulation of reagents and their subsequent encapsulation into double emulsions. Sci Rep. 2018;8(1):8143.

32. Pessi J, Santos HA, Miroshnyk I, JoukoYliruusi, Weitz DA, Mirza S. Microfluidics-assisted engineering of polymeric microcapsules with high encapsulation efficiency for protein drug delivery. Int J Pharm. 2014;472(1-2):82-87.

33. Mitropoulos AN, Perotto G, Kim S, Marelli B, Kaplan DL, Omenetto FG. Synthesis of silk fibroin micro- and submicron spheres using a co-flow capillary device. Adv Mater. 2014;26(7):1105-1110.

34. Chen J, Clay N, Kong H. Non-spherical particles for targeted drug delivery. Chem Eng Sci. 2015;125:20-24.

35. Geng Y, Discher DE. Hydrolytic degradation of poly(ethylene oxide)-block-polycaprolactone worm micelles. J Am Chem Soc. 2005;127(37):12780-12781.

36. Dendukuri D, Tsoi K, Hatton TA, Doyle PS. Controlled synthesis of nonspherical microparticles using microfluidics. Langmuir: the ACS Journal of Surfaces and Colloids. 2005;21(6):2113-2116.

37. Dendukuri D, Pregibon DC, Collins J, Hatton TA, Doyle PS. Continuousflow lithography for high-throughput microparticle synthesis. Nat Mater. 2006;5(5):365-369.
38. Habibi N, Kamaly N, Memic A, Shafiee H. Self-assembled peptidebased nanostructures: smart nanomaterials toward targeted drug delivery. Nano Today. 2016;11(1):41-60.

39. Bai S, Debnath S, Gibson K, et al. Biocatalytic self-assembly of nanostructured peptide microparticles using droplet microfluidics. Small. 2014;10(2):285-293.

40. Simon JR, Carroll NJ, Rubinstein M, Chilkoti A, López GP. Programming molecular self-assembly of intrinsically disordered proteins containing sequences of low complexity. Nat Chem. 2017;9(6): 509-515.

41. Arnon ZA, Vitalis A, Levin A, et al. Dynamic microfluidic control of supramolecular peptide self-assembly. Nat Commun. 2016;7:13190.

42. Tamayol A, Akbari M, Annabi N, Paul A, Khademhosseini A, Juncker D. Fiber-based tissue engineering: progress, challenges, and opportunities. Biotechnol Adv. 2013;31(5):669-687.

43. Haynl C, Hofmann E, Pawar K, Förster S, Scheibel T. Microfluidicsproduced collagen fibers show extraordinary mechanical properties. Nano Lett. 2016;16(9):5917-5922.

44. Konwarh R, Gupta P, Mandal BB. Silk-microfluidics for advanced biotechnological applications: a progressive review. Biotechnol Adv. 2016;34(5):845-858.

45. Peng Q, Zhang Y, Lu L, et al. Recombinant spider silk from aqueous solutions via a bio-inspired microfluidic chip. Sci Rep. 2016;6: 36473 .

46. Kinahan ME, Filippidi E, Köster S, et al. Tunable silk: using microfluidics to fabricate silk fibers with controllable properties. Biomacromolecules. 2011;12(5):1504-1511.

47. Luo J, Zhang L, Peng Q, et al. Tough silk fibers prepared in air using a biomimetic microfluidic chip. Int J Biol Macromol. 2014;66:319-324.

48. Nisisako T, Torii T. Microfluidic large-scale integration on a chip for mass production of monodisperse droplets and particles. Lab Chip 2008;8(2):287-293.
Biologics: Targets and Therapy

\section{Publish your work in this journal}

Biologics: Targets and Therapy is an international, peer-reviewed journa focusing on the patho-physiological rationale for and clinical application of Biologic agents in the management of autoimmune diseases, cancers or other pathologies where a molecular target can be identified. This journal is indexed on PubMed Central, EMBase, and Scopus.

\section{Dovepress}

The manuscript management system is completely online and includes a very quick and fair peer-review system, which is all easy to use. Visit http://www.dovepress.com/testimonials.php to read real quotes from published authors. 